Table 2. Comparison between F-SS-2 and ISSF Score

\begin{tabular}{|c|c|c|c|c|c|c|}
\hline & & \multicolumn{4}{|c|}{$\begin{array}{l}\text { ISSF Score } \\
(n=160)\end{array}$} & \multirow[b]{2}{*}{$p^{a}$} \\
\hline & & Mild & Intermediate & Severe & Total & \\
\hline F-SS-2 & Mild & $37(62.7)$ & 20 (33.9) & $2(3.4)$ & $59(100.0)$ & $<0.001$ \\
\hline Score & Intermediate & $22(53.7)$ & $17(41.5)$ & $2(4.9)$ & $41(100.0)$ & \\
\hline \multirow[t]{2}{*}{$(n=160)$} & Severe & 8 (13.3) & $43(71.7)$ & $9(15.0)$ & $60(100.0)$ & \\
\hline & Total & $\begin{array}{c}67 \\
(100.0)\end{array}$ & $80(100.0)$ & $\begin{array}{c}13 \\
(100.0)\end{array}$ & $\begin{array}{c}160 \\
(100.0)\end{array}$ & \\
\hline
\end{tabular}

F-SS-2: The second set of Familial Mediterranean Fever severity score, ISSF: International Severity Scoring System for Familial Mediterranean Fever, ${ }^{\text {apearson }}$ Chi-Square test

Conclusion: Disease severity scores should be used for treatment choice, evaluation of disease prognosis and classify patients according to disease severity in clinical trials. In the current study, we found a difference between the classifications of disease severity, which could be a result of each scoring system uses different parameters of the disease. Disclosure of Interests: None declared DOI: 10.1136/annrheumdis-2019-eular.3691

\section{THU0588 CLINICAL PHENOTYPES OF IGG4-RELATED DISEASE IN SPAIN}

María Toledano-Macías ${ }^{1}$, Andreu Fernandez-Codina ${ }^{2}$, lago Carballo ${ }^{3}$, Borja De Miguel-Campo ${ }^{4}$, Luis Sáez-Comet ${ }^{5}$, José Hernández-Rodríguez ${ }^{6}$,

Blanca Pinilla ${ }^{1}$, Fernando Martínez-Valle ${ }^{7}$, Registro Español de Enfermedad Relacionada con la lgG4 (REERIGG4) GEAS/SEMI. 'Gregorio Marañón Hospital, Internal Medicine, Madrid, Spain; ${ }^{2}$ Western University, Rheumatology and Internal Medicine, London, Canada; ${ }^{3}$ Hospital Clinico Universitario de Santiago, Medicina Interna, Santiago de Compostela, Spain; ${ }^{4}$ Hospital 12 de Octubre, Internal Medicine, Madrid, Spain; ${ }^{5}$ Hospital Universitario Miguel Servet, Internal Medicine, Zaragoza, Spain; ${ }^{6}$ Hospital Clinic, Autoimmune Diseases, Barcelona, Spain;

${ }^{7}$ Hospital Universitari Vall d'Hebron, Internal Medicine, Barcelona, Spain

Background: Recently, several clinical phenotypes in IgG4-related disease have been described in a multinational and ethnically variate cohort ${ }^{1}$.

Objectives: To assess the clinical presentation of lgG4-related disease (IgG4-RD) in Spanish patients and assess the distribution among different clinical phenotypes.

Methods: Clinical data were obtained from the Spanish IgG4-RD registry (REERIGG4) from October 2013 to December 2018, including 9 centers. We reviewed demographic data and organ involvement. The assignation of clinical phenotypes was done by 2 experts, based on organ involvement and clinical manifestations, following Wallace et al. ${ }^{1}$ subsets. The phenotypes were: pancreato-hepato-biliary (HBP), retroperitoneum and aorta (RA), head and neck limited (HNL) and Mikulicz and systemic (MS). A fifth group designated not defined (ND), included the patients that did not fit in the previous phenotypes.

Results: One-hundred patients were included. Thirty-four (34\%) were females, median age at diagnosis was 54.8 years (IQR 20.7). The ethnicity of the participants was: Caucasian $83 \%$, Hispanic $12 \%$ and NorthAfrican/Middle-East $5 \%$. Ninety-two percent were diagnosed with a biopsy. Regarding the diagnostic criteria, $85 \%$ met consensus pathology criteria and $94 \%$ comprehensive criteria.

Fifty-one patients (51\%) had systemic lgG4-RD involving $>1$ tissue. The most commonly involved tissues were: retroperitoneum (35\%), lymph nodes $(19 \%)$, orbit pseudotumor (18\%), salivary glands (16\%) and pancreas (14\%). Forty-two patients $(42 \%)$ had elevated serum IgG4.

The representation of each clinical phenotype was: HBP $14 \%$, RA $25 \%$, HNL $26 \%$, MS $20 \%$, ND $15 \%$. Patients were equally distributed from the perspective of ethnicity. Men were predominant in all groups $(71,84,70$, $73 \%$ ) except in HNL (61\% for women). Systemic disease was present in all the MS patients, but in $1 / 3$ of the other groups. Serum IgG4 was elevated in $86 \%$ of the cases in $\mathrm{HBP}, 28 \%$ in RA, $19 \%$ in $\mathrm{HNL}, 60 \%$ in MS and $40 \%$ in ND.

Conclusion: The Spanish IgG4-RD population was mainly ethnically Caucasian. Few patients had serum IgG4 elevation. The most frequent phenotype was HNL, followed by RA. The HBP phenotype was less frequent than in previous reports. The influence of race could modify the clinical expression of IgG4-RD. Knowing the regional phenotypes of IgG4-RD may help clinicians improve disease management.
REFERENCES:

[1] Wallace ZS, Zhang Y, Perugino CA, et al. Clinical phenotypes of IgG4related disease: an analysis of two international cross-sectional cohorts. Ann Rheum Dis. doi: 10.1136/annrheumdis-2018-214603

Disclosure of Interests: None declared

DOI: 10.1136/annrheumdis-2019-eular.3725

\section{THU0589 FREQUENCY, CHARACTERISTICS AND CLINICAL DETERMINANTS OF "PRODROME" IN FAMILIAL MEDITERRANEAN FEVER PATIENTS}

Hakan Babaoglu, Nuh Atas, Ozkan Varan, Hasan Satıș, Reyhan Bilici Salman, Aslıhan Avanoglu Güler, Hazan Karadeniz, Berna Goker, Seminur Haznedaroglu, Mehmet Akif Ozturk, abdurrahman tufan. Gazi University Faculty of Medicine, Department of Internal Medicine-Rheumatology, Ankara, Turkey

Background: Prodrome was defined by the presence of manifestations that precede an FMF attack and predict its emergence. This period might be turned to a window of the opportunity for the prompt treatment of impending attacks with fast-acting IL-1 inhibitors.

Objectives: We aimed to determine the frequency, characteristics and clinical determinants of prodrome in patients with FMF

Methods: 401 FMF patients were enrolled in this cross-sectional study. We applied a questionnaire to the FMF patients about attack types, preattack manifestations and latent time. Four hours was accepted as the cutoff point for prodrome as based on the literature, which is compatible with the pharmacokinetics of anakinra.

Results: The mean age was $37.7( \pm 11.0)$ years, and the disease duration was 20 years (3-58). 248 of the patients $(61.8 \%)$ were female. MEFV gene mutations were achieved in $364(90.8 \%)$ patients, of these $121(30.2 \%)$ patients harbored homozygous exon 10 mutations. Patients with a latent time over four hours considered as prodrome positive (PP) patients $(n=141)$. Male gender, homozygous MEFV mutations, peritonitis, pleuritis, arthritis and ELE were more pronounced in PP group. $25.9 \%$ of patients with peritonitis, $14.7 \%$ of patients with pleuritis, $13.2 \%$ with arthritis and $5.5 \%$ with ELE had prodrome with variable latent time durations (median, 6.7(4-72), 6 (4-48), 6 (4-72), and 7.7 (4-30) hours, respectively). Prodrome was found to be more common in those with peritonitis. Male gender, having peritonitis or arthritis were found to be the independent clinical determinants of having prodrome (RR 1.72 (1.07-2.76), $P=0.02$, 4.27 (1.80-10.1), $P=0.001,1.77$ (1.04-3.04), $P=0.04$, respectively). Age, MEFV mutations, pleuritis and ELE were not found as clinical determinants of prodrome.

Conclusion: Especially male patients with peritonitis or arthritis tend to have prodrome more than other patients, and should be questioned. Prodrome positive patients are candidates for prevention of the impending attacks with on-demand treatments.

Table 1. Patients demographics and disease characteristics

\begin{tabular}{lccc}
\hline & $\begin{array}{c}\text { Prodrome } \\
(+) \\
(\mathbf{n = 1 4 1 )}\end{array}$ & $\begin{array}{c}\text { Prodrome }(-) \\
(\mathbf{n}=260)\end{array}$ & $\mathbf{p}$ \\
\hline Age, years & $37.7(10.5)$ & $37.8(12.8)$ & 0.92 \\
Female & $78(55.3 \%)$ & $170(65 \%)$ & 0.05 \\
Age at FMF diagnosis, & $24(3-62)$ & $25(2-59)$ & 0.52 \\
years & & & \\
Disease duration, years & $21(4-58)$ & $19(3-58)$ & 0.08 \\
Fever & 0 & $9(3.5 \%)$ & 0.03 \\
Peritonitis & $133(94.3 \%)$ & $197(75.8 \%)$ & $<0.001$ \\
Pleuritis & $92(65.2 \%)$ & $123(47.3 \%)$ & 0.01 \\
Arthritis & $103(72.9 \%)$ & $129(49.8 \%)$ & $<0.001$ \\
Myalgia & 0 & $6(2.3 \%)$ & 0.09 \\
Erysipelas like erythema & $46(32.6 \%)$ & $58(22.3 \%)$ & 0.02 \\
Homozygous* & $57(43.2 \%)$ & $64(27.6 \%)$ & 0.02 \\
M694V/M694V & $50(35.5 \%)$ & $51(19.6 \%)$ & 0.01 \\
M694V/- & $19(13.5 \%)$ & $46(17.7 \%)$ & 0.19 \\
M694V/M680I & $13(9.2 \%)$ & $23(8.8 \%)$ & 0.98 \\
M694V/V726A & $12(8.5 \%)$ & $20(7.7 \%)$ & 0.88 \\
M694V/E148Q & $6(4.3 \%)$ & $15(5.8 \%)$ & 0.45 \\
M680I/M680I & $6(4.3 \%)$ & $9(3.5 \%)$ & 0.76 \\
\hline * 364 Pationts MEFV mutations & & & \\
\hline
\end{tabular}

* 364 patients MEFV mutations were achieved. $9.2 \%$ of patients MEFV mutations is not known, $7.7 \%$ of patients is gen negative FMF.

Acknowledgement: none

Disclosure of Interests: None declared

DOI: 10.1136/annrheumdis-2019-eular.6550 
Okan Aydin, Serdal Ugurlu, Huri Ozdogan. Cerrahpasa Medical Faculty, University of Istanbul - Cerrahpasa, Division of Rheumatology, Department of Internal Medicine, Istanbul, Turkey

Background: Familial Mediterranean fever (FMF) is a disease with an onset before 20 years of age in $90 \%$ of the patients. However late onset FMF defined as age of onset over 40 years is being recognised more frequently.

Objectives: To better define patients with FMF who had their first attack before age 40 and compare them with early onset patient group in Turkish population

Methods: The files of 2180 FMF patients followed in a single center between 2008-2017 who have fulfilled Tel-Hashomer criteria, were reviewed with regard to age of onset 40 years and over (index patients, Group 1). For control purposes files before and after the index patients were browsed and first patients with an onset before age 20 years (Group 2) were included. The demographic, clinical and genetic characteristics are compared between these 2 subgroups.

Results: Patients with an onset after 40 years consisted $2.7 \%$ of our FMF population. 50 of the 59 patients with an onset 40 years or over were re-evaluated and compared with early onset group consisting of 100 patients (Table 1). The delay in diagnosis, and disease duration were significantly longer and number of patients with M694V homozygosity and M694V allele frequency were significantly more frequent among group 2. In general, phenotypes of both onset groups were similar, the only significant differences being the frequency of fever and myositis which were less common among group 1. Also response to colchicine was more pronounced in group 1. One other interesting observation was the low incidence of amyloidosis in a group with such a significant delay in diagnosis and thus treatment.

Table 1. Demographic, clinical and genetic features of the study groups

\begin{tabular}{|c|c|c|c|}
\hline & $\begin{array}{c}\geq 40 \text { years } \\
\quad n=50\end{array}$ & $\begin{array}{c}\leq 20 \text { years } n= \\
100\end{array}$ & $\mathrm{p}$ \\
\hline Sex $(F: M) ;$ present age (mean $\pm S D)(y r)$ & $32: 18 ; 57.2 \pm 7.9$ & $62: 38 ; 31.8 \pm 9.1$ & $\begin{array}{l}\mathrm{NS} ; \\
<0.001\end{array}$ \\
\hline Mean age at onset,(mean $\pm s d)(y r)$ & $45.6 \pm 5.2$ & $8.7 \pm 4.8$ & $<0.001$ \\
\hline $\begin{array}{l}\text { Mean age at diagnosis (mean } \pm s d) \\
(y r)\end{array}$ & $50.4 \pm 7.3$ & $19.1 \pm 11.2$ & $<0.001$ \\
\hline Delay in diagnosis (mean $\pm s d$ ) (yr) & $4.8 \pm 5.5$ & $10.4 \pm 11.8$ & $<0.001$ \\
\hline $\begin{array}{l}\text { Mean disease duration (mean } \pm s d \text { ) } \\
\text { (yr) }\end{array}$ & $11.5 \pm 6.4$ & $23.1 \pm 10.8$ & $<0.001$ \\
\hline Abdominal pain, $n(\%)$ & $44(88)$ & $89(89.0)$ & NS \\
\hline Chest pain, $\mathrm{n}(\%)$ & $7(14.0)$ & $27(27.0)$ & NS \\
\hline Fever, $\mathbf{n}(\%)$ & $30(60.0)$ & $81(81.0)$ & 0.005 \\
\hline Arthritis, n (\%) & $12(24.0)$ & $33(33.0)$ & 0.25 \\
\hline Myalgia, n (\%) & $1(2.0)$ & $12(12.0)$ & 0.04 \\
\hline Amyloidosis, n (\%) & $1(2.0)$ & $3(3.0)$ & NS \\
\hline Positive family history, $\mathrm{n}(\%)$ & $33(68.7)$ & $62(65.2)$ & NS \\
\hline Response to colchicine, $\mathrm{n}(\%)$ & $37(82.2)$ & $93(94.8)$ & 0.014 \\
\hline M694VHomozygous, n (\%) & 2(4.5) & $23(25.8)$ & 0.003 \\
\hline $\mathrm{N}$ of M694Vallelles & $24(48)$ & $82(82)$ & 0.014 \\
\hline No mutation, n (\%) & $3(6.8)$ & $2(2.2)$ & NS \\
\hline
\end{tabular}

Conclusion: FMF should be included among the differential diagnosis of patients over 40 years of age with recurrent autoinflammatory manifestations. Less than $3 \%$ of FMF patients experience their first attacks after 40 years of age. The frequency of $\mathrm{M} 694 \mathrm{~V}$ is significantly less in the late onset group, pointing out a milder disease.

Disclosure of Interests: None declared

DOI: 10.1136/annrheumdis-2019-eular.6560

\section{THU0591}

SALIVARY GLAND INVOLVEMENT DISPARITIES IN CLINICAL AND LABORATORY CHARACTERISTICS OF IGG4-RELATED DISEASE: A RETROSPECTIVE STUDY OF 428 PATIENTS

Yanying Liu ${ }^{1}$, Miao Xue ${ }^{2}$, Zhenfan Wang ${ }^{1}$, Qiaozhu Zeng ${ }^{1}$, Limin Ren ${ }^{1}$,

Yanyan Zhang ${ }^{3}$, Shanshan Zhang ${ }^{4}$, Yi Wang ${ }^{5}$, Danhua Shen ${ }^{6}$, Changsheng Xia ${ }^{7}$, Guangyan $\mathrm{Yu}^{3}$, LI Zhan-Guo ${ }^{1} .{ }^{1}$ Peking University People's Hospital,

Rheumatology and Immunology, Beijing, China; ${ }^{2}$ The First Hospital of Lanzhou

University, Rheumatology and Immunology, Lanzhou Shi, China; ${ }^{3}$ Peking

University School of Stomatology, Oral and Maxillofacial Surgery, Beijing, China;

${ }^{4}$ Peking University People's Hospital, Ultrasound, Beijing, China; ${ }^{5}$ Peking

University People's Hospital, Radiology, Beijing, China; ${ }^{6}$ Peking University

People's Hospital, Pathology, Beijing, China; ${ }^{7}$ Peking University People's Hospital, Clinical Laboratory, Beijing, China

Background: IgG4 related disease (IgG4-RD) is recently recognized as a fibro-inflammatory condition featured by tumefactive lesions in multiple organs, among which salivary gland is one of the most commonly involved sites. Some previous studies have suggested that salivary gland involved IgG4-RD (IgG4-RD SG+) patients may present different features compared to IgG4-RD patients without salivary gland lesions (IgG4-RD SG-), however, only dozens of patients were examined and the comparison has not been studied in detail.

Objectives: We undertook this study to compare detailed demographic, clinical and laboratory characteristics of IgG4-RD patients with salivary gland lesion (IgG4-RD $\mathrm{SG}+$ ) and salivary gland free IgG4-RD (IgG4-RD SG-) in a large cohort.

Methods: We carried out a retrospective review of the medical records of 428 cases of IgG4-RD diagnosed at Peking University People's hospital between March 2006 and May 2018 .

Results: Among 428 patients, 249 had salivary gland affected. IgG4-RD $\mathrm{SG}+$ patients showed younger age at disease onset and diagnosis, and a longer interval between symptom onset and diagnosis. IgG4-RD SG+ group involved more female patients, and allergic diseases were more common in this group. In terms of organ involvement, IgG4-RD SG+ group were more frequently presented with lacrimal gland involvement, while lymph node, retroperitoneal fibrosis, pancreas, biliary system, kidney, and aorta were more prominent in IgG4-RD SG- group. In addition, the serum IgG4 levels, IgG4/lgG ratio and IgE level were significantly higher in IgG4-RD SG+ patients. Patients with eosinophilia were more common in IgG4-RD SG+ group, while elevated ESR, CRP, and positive ANA were more common in IgG4-RD SG- group.

Conclusion: We have revealed demographic, clinical and laboratory differences between IgG4-RD SG+ and SG- patients, which indicated potential differences in pathogenesis and important implications for the diagnosis and management of these two phenotypes.

\section{REFERENCES:}

[1] Kamisawa T, Zen Y, Pillai S, Stone JH. IgG4-related disease. Lancet. 2015;385(9976):1460-71

[2] Moriyama M, Ohta M, Furukawa S, Mikami Y, Tanaka A, Maehara T, et al. The diagnostic utility of labial salivary gland biopsy in IgG4-related disease. Mod Rheumatol. 2016;26(5):725-9.

[3] Higgs BW, Liu Y, Guo J, Sebastian Y, Morehouse C, Zhu W, et al. Highthroughput RNA sequencing reveals distinct gene signatures in active IgG4-related disease. Sci Rep. 2017;7(1):17567.

[4] Inoue D, Yoshida K, Yoneda N, Ozaki K, Matsubara T, Nagai K, et al. IgG4-related disease: dataset of 235 consecutive patients. Medicine (Baltimore). 2015;94(15):e680.

Disclosure of Interests: None declared

DOI: 10.1136/annrheumdis-2019-eular.3690

\section{THU0592 PREDICTORS OF RELAPSE OF IGG4-RELATED DISEASE AFTER INDUCTION THERAPY: A RETROSPECTIVE STUDY}

Tomohiro Yoshida, Keisuke Nishimura, Kaoru Mizukawa, Keiichiro Kadoba, Hiroki Mukoyama, Daisuke Waki, Toshihiko Yokota, Hiroyuki Murabe. Kurashiki Central Hospital, Department of Endocrinology and Rheumatology, Kurashiki, Okayama, Japan

Background: IgG4-related disease (IgG4-RD) is a systemic fibroinflamma tory condition characterized by tumefactive lesions and, often but not always, elevated serum lgG4 levels [1]. Glucocorticoids (GC) are usually used as induction therapy for IgG4-RD. However, relapse often occurs following GC tapering, and predictors of relapse remain unclear. 